\title{
DIRECT NUMERICAL SIMULATION OF A STRAIGHT VORTEX TUBE IN A LAMINAR BOUNDARY-LAYER FLOW
}

\author{
K. MATSUURA \\ Graduate School of Science and Engineering, Ehime University, Japan.
}

\begin{abstract}
Effects of circulation on the evolution of vortex tubes and the associated response of near-wall flows in the shear of laminar boundary-layer flows are investigated using a model proposed by Hon and Walker (Hon, T.L. \& Walker, J.D.A, Computers \& Fluids, 20(3), pp. 343-358, 1991). Direct numerical simulations with freestream Mach number of 0.5 are conducted. Firstly, the dynamics of single hairpin vortex is investigated. Numerous secondary hairpin vortices, much more than previously reported, which are regularly aligned in the streamwise direction are allowed to be newly generated according to the shear-layer instability of the legs of an initial hairpin vortex. Small-scale turbulence is then produced when the circulation is sufficiently large. Secondly, a straight vortex tube model is investigated. Sinuous deformation of a shear layer, which leads to the generation of discrete hairpin vortices, becomes obvious especially near the upper region of the vortex tube. In order to quantify the initial instability triggering the generation of the secondary hairpin vortices, quasi-linear stability analysis is conducted. While only one unstable mode appears when the circulation is small, two modes, that is, off-wall mode and near-wall mode, appear when the circulation is large. The cases of circulation where the two modes appear correspond to those of circulation where the production of small-scale turbulence is observed in the simulations of the single hairpin vortex.
\end{abstract}

Keywords: boundary layer, direct numerical simulation, hairpin vortex, laminar-turbulent transition, stability, turbulence, vortex tube.

\section{INTRODUCTION}

A hairpin vortex is considered as the basic building block of turbulence near a solid wall [1]. Since the work of Theodorsen [2], there have been many studies on hairpin/horseshoe vortices. Formation of the hairpin vortices has been discussed in many literatures [3-8]. Among previous studies is that of Moin et al. [9] who studied the deformation of a hairpin-shaped vortex filament under self-induction and in the presence of shear numerically using the BiotSavart law, showed a mechanism for the generation of ring vortices in turbulent shear flows. Acarlar and Smith [10] visualized the dynamics of hairpin vortices in the downstream wake of a hemispheric obstacle. Hon and Walker [11] developed a numerical method based on the Lagrangian vortex method that allows the accurate computation of the trajectory of a three-dimensional vortex having a small core radius. Using this method, they showed that a two-dimensional vortex containing a small three-dimensional disturbances distorts into a complex shape with subsidiary hairpin vortices forming outboard of the original hairpin vortex. Singer et al. [12] studied the formation and growth of a hairpin vortex in a flat-plate boundary layer and its later development into a young turbulent spot. Initial hairpin vortex was triggered by fluid injection through a slit in the wall. They reported the development of multiple hairpin vortex heads between stretched legs, the formation of new vortices beneath the streamwise-elongated vortex legs and the formation of a travelling region of highly disturbed flow with an arrowhead shape similar to that of a turbulent spot. Zhou et al. $[13,14]$ studied, by direct numerical simulation (DNS), the evolution of a symmetric pair of quasistreamwise vortical structures extracted from the two-point correlation tensor of turbulent channel flow data by a linear stochastic estimation procedure. They observed that sufficiently 
strong hairpin vortices generate a hierarchy of secondary hairpin vortices, and the mechanism of their creation closely resembles the formation of the primary hairpin vortex. Liu et al. [15] conducted a compressible DNS for non-linear stages of laminar-turbulent transition. They discussed the coherent vortex structure appearing in the late stages of transition and the mechanism of the formation of single vortex ring, multiple vortex rings, and small length scale. At the inflow, they assumed two-dimensional waves and a pair of oblique waves in addition to the laminar boundary layer profile to reproduce transition of K-regime. Duguet et al. [16] investigated the region of phase space separating transitional from relaminarizing trajectories regarding the Blasius boundary layer, and a quasi-cyclic mechanism for the generation of hairpin vortex offspring. Cohen et al. [17] proposed a model consisting of minimal flow elements that can produce packets of hairpins. The three components of the model are simple shear, a counter-rotating vortex pairs having finite streamwise vorticity magnitude and a two-dimensional wavy (in the streamwise direction) spanwise vortex sheet. Eitel-Amor et al. [18] studied the characteristics of hairpin vortices in turbulent boundary layers by parallel and spatially developing simulations. They found that secondary hairpins are only created shortly after initialization, with all rotational structures decaying for later times. They also reported that the regeneration process is rather short-lived and may not sustain once a turbulent background is developed. Sabatino et al. [19] studied experimentally hairpin vortex formation in a laminar boundary layer by fluid injection through a narrow slot. They discussed hairpin vortex head, legs and secondary hairpin vortex focusing on its circulation strength. Although there are many studies on hairpin vortices, effects of changing quantities associated with the hairpin vortices on its stability and near-wall dynamics have not been necessarily investigated systematically. In this regard, the hairpin model proposed by Hon and Walker [11] is particularly interesting because it has degrees of freedom for the circulation, size, the angle-to-wall and the core radius of a hairpin vortex, and will directly enable such systematic investigations. However, the model has not been studied in detail within the framework of DNS. So, in this study, by DNS, the evolution of vortex tubes in the shear of background laminar boundary-layer flows and its response of near-wall flows using the single hairpin vortex model proposed by Hon and Walker [11] and a straight vortex tube which corresponds to the leg portion of the model is investigated. We focus on the effects of circulation. In Section 2, the present computational methods are described. In Section 3, DNS of the single hairpin vortex and straight vortex tube are conducted. By using the results of the straight vortex tube, quasi-linear stability analysis is conducted to quantify the initial instability triggering the generation of the secondary hairpin vortices. In Section 4, conclusions for this study are drawn.

\section{COMPUTATIONAL DETAILS}

Freestream Mach number is 0.5 and streamwise Reynolds number at the inlet is $5.34 \times 10^{5}$. At the initial time $t=0$, a single hairpin vortex or a straight vortex tube of finite length is embedded in a laminar boundary layer. The vortex axes of the legs of the hairpin vortex and the straight vortex tube are inclined to the $\mathrm{x}$ (streamwise) axis and its angle is $4^{\circ}$. Computation is impulsively started from the initial condition. Three kinds of circulation magnitudes are considered in this study. The circulations of the vortex $\Gamma /(2 \pi)$ non-dimensionalized by freestream velocity and the displacement thickness $\delta_{\text {in }}{ }^{*}$ at the inlet, denoted as $\Gamma^{*}$, is chosen as 6.24, 12.4 and 24.9. Velocity fields induced by the vortex tubes are generated by the algorithm of Hon and Walker [11] explained by the eqns (1)-(3). The algorithm is a modification of the Moore's algorithm [20], which is reported to exhibit strong numeri- 
cal instability for small value of a core radius $a$. At an arbitrary location in space $\boldsymbol{X}_{0}$, the velocity field $\boldsymbol{u}$ due to a vortex is basically described by the Biot-Savart Law. Contour $C$ is a curve defining a vortex tube, and $\Gamma$ is circulation about the vortex core, and $\boldsymbol{u}_{\text {ext }}$ is a background velocity field.

$$
\boldsymbol{u}\left(\boldsymbol{X}_{0}\right)=-\frac{\Gamma}{4 \pi} \int_{C} \frac{\left(\boldsymbol{X}_{0}-\boldsymbol{X}\right)}{\left|\boldsymbol{X}_{0}-\boldsymbol{X}\right|^{3}} \times d \boldsymbol{X}+\boldsymbol{u}_{\text {ext }}\left(\boldsymbol{X}_{0}\right),
$$

When $\boldsymbol{X}_{0}$ is close to the curve $C$, the above equation is switched to the following equation to prevent singularity. The parameter $s$ is a Lagrangian coordinate which ranges from $-\infty$ to $\infty$ along the vortex. The integrand of $R\left(s, s_{0}\right)$ is shown in [11].

$$
\begin{gathered}
\boldsymbol{u}\left(s_{0}\right)= \\
\frac{\Gamma}{4 \pi} \int_{C} R\left(s, s_{0}\right) d s+\frac{\Gamma}{4 \pi}\left(\frac{\partial \boldsymbol{X}}{\partial s}\right)_{0} \times\left(\frac{\partial^{2} \boldsymbol{X}}{\partial s^{2}}\right)_{0} \int_{C} P(s) d s+\boldsymbol{u}_{\text {ext }}, \\
P(s)=\frac{\frac{1}{2}\left(s-s_{0}\right)^{2}}{\left[\left(s-s_{0}\right)^{2}\left(\frac{\partial \boldsymbol{X}}{\partial s}\right)_{0}^{2}+\mu^{2}\right]^{3 / 2}}, \\
\mu=\exp (-3 / 4) a, \quad a=0.25
\end{gathered}
$$

The initial vortex configuration used in the case of the single hairpin vortex is of the form

$$
\boldsymbol{X}(s)=A(\boldsymbol{i} \cos \alpha+\boldsymbol{j} \sin \alpha) \exp \left(-\beta s^{2}\right)+\boldsymbol{j}+\boldsymbol{s} \boldsymbol{k}
$$

Here, $\boldsymbol{i}, \boldsymbol{j}, \boldsymbol{k}$ are unit vectors corresponding to the streamwise $(x)$, normal $(y)$ and spanwise (z) directions, respectively. Equation (4) represents a two-dimensional vortex located a unit distance from the wall with a three-dimensional distortion which is symmetrical about $s=0$. In addition, $A$ represents the amplitude of the distortion and $\alpha$ is the angle that the plane of the distortion makes with the wall. $\beta$ is a (large) number determining the effective spanwise width of the initial distortion. The initial configuration used in the case of the straight vortex tube is

$$
\boldsymbol{X}(s)=A s(i)\left(\begin{array}{c}
\cos \varphi \\
\sin \varphi \\
0
\end{array}\right)+\left(\begin{array}{l}
x_{c} \\
y_{c} \\
z_{c}
\end{array}\right)
$$

Here, $A$ is $24 \delta_{\text {in }}{ }^{*} \cdot\left(x_{\mathrm{c}}, y_{\mathrm{c}}, z_{\mathrm{c}}\right)$ is the root position of the vortex tube. $x_{\mathrm{c}}$ is $30 \delta_{\text {in }}{ }^{*}$ downstream of the inlet and $y_{\mathrm{c}}=0.4 \delta_{\text {in }}{ }^{*} \cdot \varphi$ is an angle of inclination to the wall and $\varphi=4^{\circ}$. The values of $A$ and $\varphi$ are determined by conducting the same simulation on transition as [15] separately. The governing equations are the unsteady three-dimensional compressible Navier-Stokes equations. To close the system the perfect gas law is assumed. The equations are solved by the sixth-order finite-difference method. Time-accurate solutions to the governing equations are obtained by the third-order Runge-Kutta scheme. The details of the present numerical method are shown in [21]. 
Regarding the boundary condition, inflow velocity and temperature profiles are specified and pressure is extrapolated from the interior at the inlet. The inflow profile is obtained by solving the boundary layer equation [22]. At the outflow and upper boundaries, nonreflecting boundary conditions with the mean static pressure $p_{\infty}=101,325 \mathrm{~Pa}$ are imposed. At the wall, non-slip, isothermal wall condition $T_{\infty}=273.15 \mathrm{~K}$ is imposed. Periodicity is imposed in the spanwise direction. Computational domains are a rectangular domain with dimensions $124.9 \delta_{\text {in }}, 16.4 \delta_{\text {in }}$ and $22.0 \delta_{\text {in }}$ in the $x, y$ and $z$ directions, respectively, for the simulations of the single hairpin vortex, and a rectangular domain with half spanwise size is used for the simulations of the straight vortex tube with $\Gamma^{*}=6.24$ and 12.4. Large domain size is basically used in the spanwise direction to reduce discontinuities in generated velocity fields due to circulation of the initial vortex tubes. Two kinds of mesh are used in this study. The number of mesh points is basically $301 \times 210 \times 128$ in the $x$ (streamwise), $y$ (wallnormal) and $z$ (spanwise) directions, respectively. The half domain mesh has the number of mesh points $301 \times 210 \times 64$. The accuracy of the computation of the straight vortex tube with $\Gamma^{*}=12.4$ is confirmed by a simulation with finer mesh with $601 \times 417 \times 128$ points.

Spanwise averaged grid resolutions $\Delta x^{+}, \Delta y^{+}{ }_{\text {min }}$ and $\Delta z^{+}$are $10.8,0.362$ and 4.49 , respectively, and locally maximum grid resolutions are $18.1,0.61$ and 7.6 , respectively.

\section{RESULTS AND DISCUSSION}

Firstly, the dynamics of a single hairpin vortex and associated response of near-wall flows are investigated using a model proposed by Hon and Walker [11]. Figures 1-3 shows time sequence of the vortex deformation and the dynamic response of the boundary layer for $\Gamma^{*}=6.24-24.9$. Vortices are visualized by the iso-surfaces of the second invariance of the velocity gradient tensor. The colour shows a velocity component in the $x$ direction divided by a sound speed. When $\Gamma^{*}=6.24$, that is, the circulation is small, hairpin vortices aligned in the streamwise direction are generated bridging over the hairpin legs. However, because the circulation is weak, the generation of the hairpin vortices does not connect to the clear appearance of turbulent regions near the wall. When $\Gamma^{*}=12.4$, that is, the circulation is medium, aligned hairpin vortices over the hairpin legs and turbulent regions consisting of hairpin vortices are generated. In earlier published literatures [1, 3, 7, 17], only a few secondary hairpin vortices are observed. Especially at $t^{*}=174$, numerous secondary hairpin vortices, much more than previously reported, which are regularly aligned in the streamwise direction, are allowed to be newly generated according to the shear-layer instability of the legs of an initial hairpin vortex. Compared with the case of $\Gamma^{*}=6.24$, streamwise distance between the hairpin vortices becomes shorter, and small-scale turbulence is then produced. When $\Gamma^{*}=24.9$, that is, the circulation is large, quasi-streamwise vortices appear near the upstream root of the legs. Generation of small-scale turbulence is much more accelerated compared with the case of $\Gamma^{*}=12.4$. Although hairpin vortices appear mainly between the legs around $t^{*}=174$, the region of hairpin vortices becomes broader in the spanwise direction. In addition to the central region sandwiched between the two legs, hairpin vortices are generated near the root of the two legs. Near the tail of the turbulent spot, the region of hairpin vortices is divided into two regions corresponding to the hairpin legs at $t^{*}=87-174$.

Secondly, the straight vortex tube model is investigated [23]. In order to analyse vorticity dynamics, the following vorticity equation [24] are considered. 


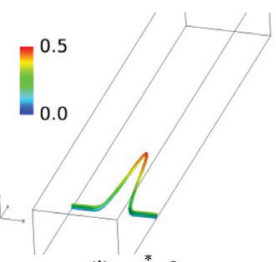

(i) $t^{*}=0$

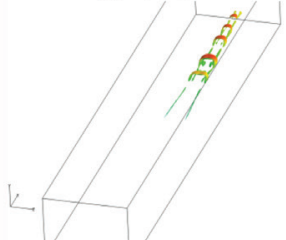

(iv) $t^{*}=217.5$

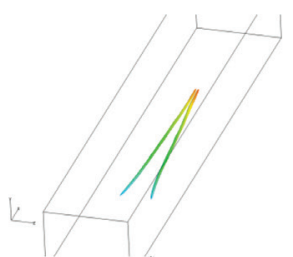

(ii) $t^{*}=87$

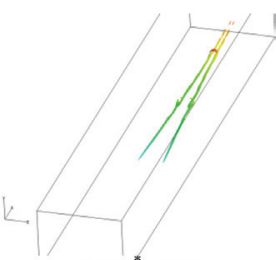

(iii) $t^{*}=174$

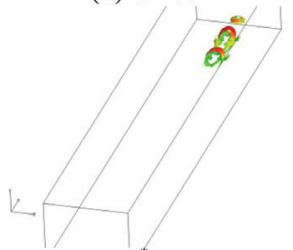

(v) $t^{*}=261$

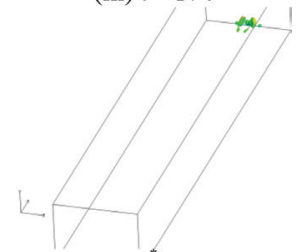

(vi) $t^{*}=348$

(a) $\Gamma^{*}=6.24$

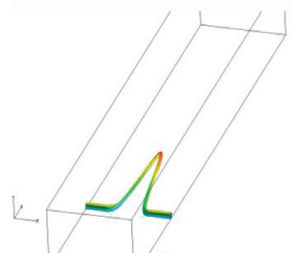

(i) $t^{*}=0$

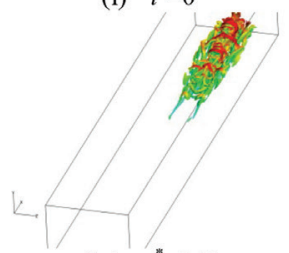

(iv) $t^{*}=261$

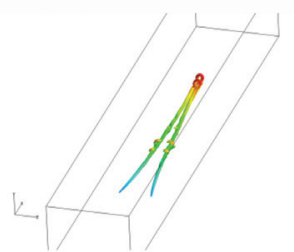

(ii) $t^{*}=87$

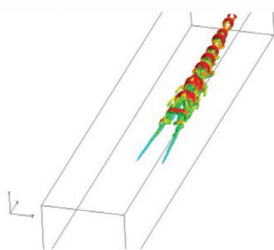

(iii) $t^{*}=174$

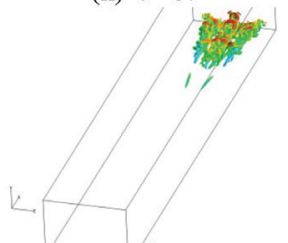

(v) $t^{*}=348$

Figure 1 ( cont' $d)$ : (b) $\Gamma^{*}=12.4$

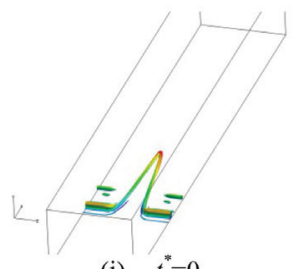

(i) $t^{*}=0$

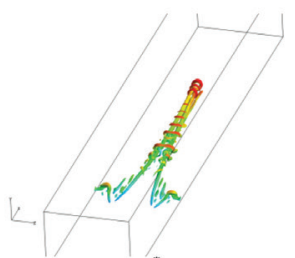

(ii) $t^{*}=87$

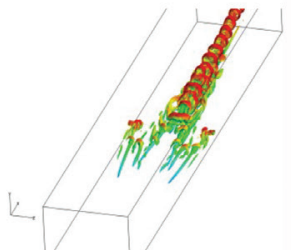

(iii) $t^{*}=174$

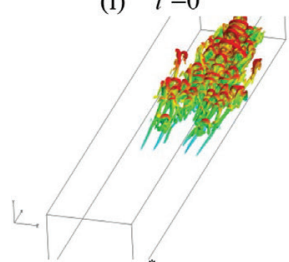

(iv) $t^{*}=261$

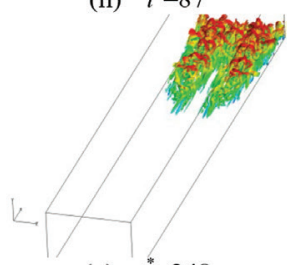

(v) $t^{*}=348$

Figure 1 (cont' $d$ ): (c) $\Gamma^{*}=24.9$

Figure 1: Time sequence of the deformation of the single hairpin vortex and the dynamic response of the boundary layer. The colour shows a streamwise velocity divided by a sound speed, that is., Mach number. (b) $\Gamma^{*}=12.4$ (c) $\Gamma^{*}=24.9$ 


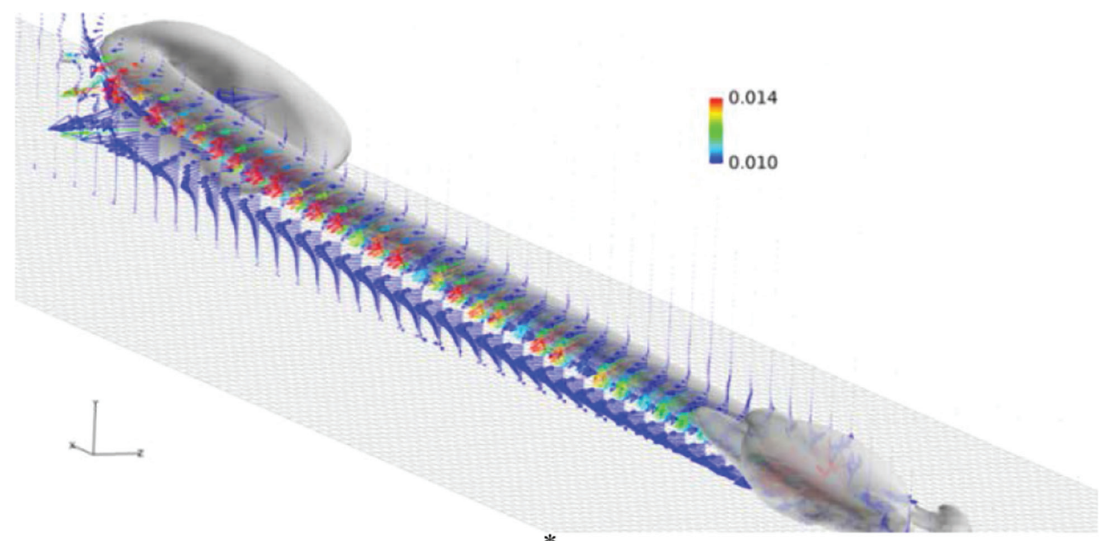

(a) $t^{*}=21.8$

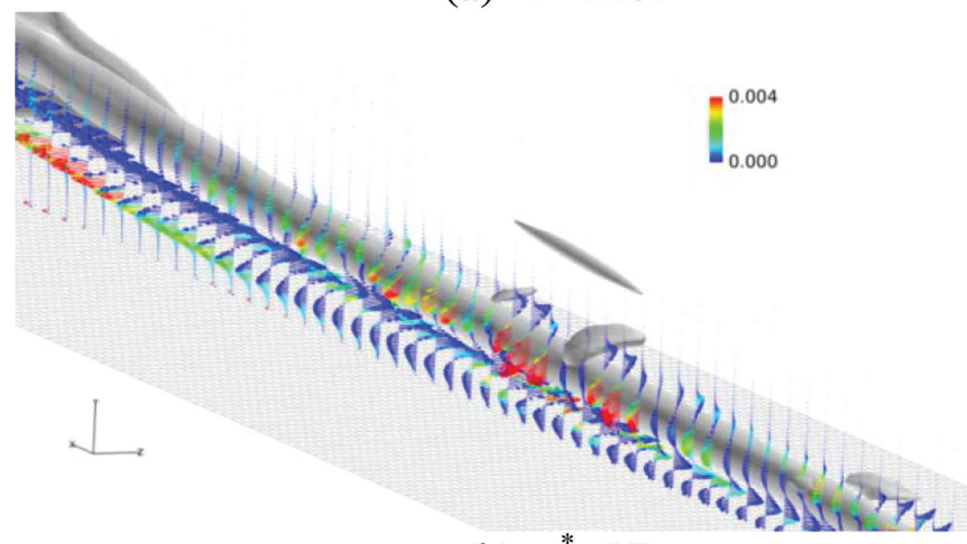

(b) $t^{*}=87$

Figure 2: Vector plot of increment of vorticity vector and instantaneous vortical structures at $t^{*}=21.8$ and 87 as a result of the simulation of the straight vortex tube with $\Gamma^{*}=12.4$.

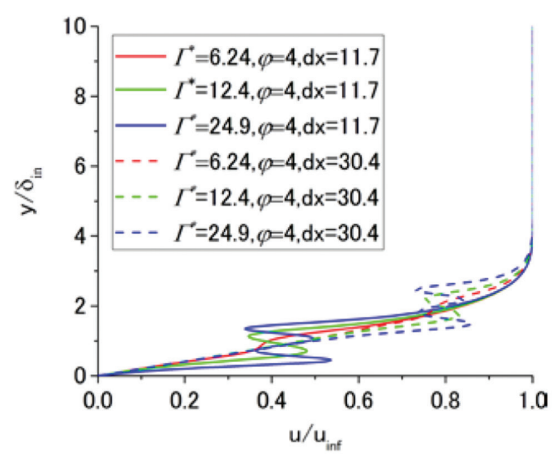

(a)

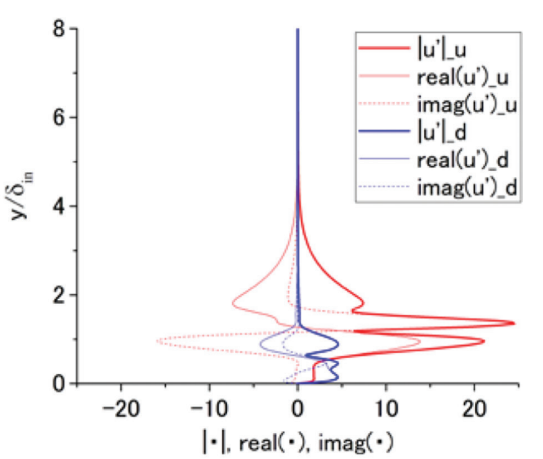

(b)

Figure 3: Mean velocity profiles used for the linear stability analysis extracted when $t^{*}=$ 21.78 (a) and absolute value, real part and imaginary part of eigenfunctions of streamwise velocity of mode- $u$ (off-wall mode) and mode-l (near-wall mode) (b) 


$$
\begin{aligned}
\frac{\partial \boldsymbol{\Omega}}{\partial t}= & -(\boldsymbol{V} \cdot \nabla) \boldsymbol{\Omega}+(\boldsymbol{\Omega} \cdot \nabla) \boldsymbol{V}+\nu \Delta \boldsymbol{\Omega}-\boldsymbol{\Omega}(\operatorname{div} \boldsymbol{V})+ \\
& \frac{\nabla \rho}{\rho} \wedge \frac{d \boldsymbol{V}}{d t}+E V V^{* *}, \\
E V V^{* *}= & \left.\frac{1}{\rho}\left(\nabla \times \boldsymbol{E}_{1}\right)-\nabla \mu \wedge \nabla \times \boldsymbol{\Omega}\right), \\
\boldsymbol{E}_{1}= & 2 \boldsymbol{S} \cdot \nabla \mu-\frac{2}{3}(\nabla \cdot \boldsymbol{V}) \nabla \mu
\end{aligned}
$$

Here, $\boldsymbol{\Omega}$ is the instantaneous vorticity vector, $\boldsymbol{V}$ is the velocity vector, $\boldsymbol{v}$ is the kinematic viscosity, $\rho$ is the density, $t$ is time and $S$ is the strain rate tensor, · is the product obtained by contraction upon the derivative coordinate. Figure 2 shows increment vectors of the vorticity vector and the instantaneous vortical structures at $t^{*}=21.8$ and 87 as a result of the simulation of the straight vortex tube with $\Gamma^{*}=12.4$. Sinuous deformation of a shear layer, which leads to the generation of discrete hairpin vortices, appears especially near the upper region of the vortex tube.

From the previous simulations of a hairpin vortex, secondary hairpin vortices over the hairpin legs are trigged by the sinuous deformation near the upper edge of the two legs, that is, shear layers created above the two quasi-streamwise vortices. In order to quantify its initial instability, linear stability analysis is conducted using the velocity profiles. Temporal stability analysis assuming disturbances of $\exp [i(\alpha x+\beta z-\omega t)]$ was conducted. After searching for candidate unstable mode by a global method, a local method with the Newton iteration is used. The procedure is described in [25]. The accuracy of the programme developed is successfully validated against the first 5 modes (phase velocity) of the compressible stability equations in the incompressible limit $\left(M=10^{-6}\right)$ shown in [25]. The present stability analysis is not necessarily strict in investigating the stability of the vortex tube in the shear. Although the vortex tube is neither parallel to the wall nor uniform in the spanwise direction, the present analysis assumes parallelism and spanwise uniformity of mean flows. In this regard, the present analysis employs a quasi-parallel approach by using boundary-layer profiles at multiple streamwise positions to cope with such non-parallel flow. In addition, characteristics of two-dimensionality, that is, events in the $x-y$ plane, are strong regarding the initial instability. Therefore, assuming the spanwise uniformity is reasonable.

Mean flows used in the stability analysis are extracted from instantaneous flow fields. Figure 3a shows the boundary layer profiles obtained at $\mathrm{d} x=11.7$ and 30.4 around the centre of the vortex tube in the spanwise direction when $t^{*}=21.75$ for $\Gamma^{*}=6.24-24.9$. Here, $\mathrm{d} x \equiv x-x_{\mathrm{c}}$. These locations correspond to those closer to the root and tip, respectively, of the vortex tube from its streamwise centre while preventing peculiarity arising from the ends of the vortex tube of finite length. Profile deformation by the vortex tube is generated near the middle of the boundary layer at $\mathrm{d} x=11.7$ and near the edge inside the boundary layer at $\mathrm{d} x$ $=30.4$. While a small step is introduced in the profile when $\Gamma^{*}=6.24$, large deformation of the magnitude about $0.17 u_{\infty}$ is introduced when $\Gamma^{*}=24.9$. When $\Gamma^{*}=6.24$ and 12.4 , there are one and two unstable modes, respectively. The two modes correspond to disturbances that amplify in the lower and upper shear layers, and are denoted as "near-wall mode (mode-l)" and "off-wall mode (mode- $u$ )", respectively. The cases of circulation where the two modes appear correspond to those where the production of small-scale turbulence is observed in the simulations of the single hairpin vortex mentioned in Figs 1and $3 \mathrm{~b}$ shows the absolute value, 


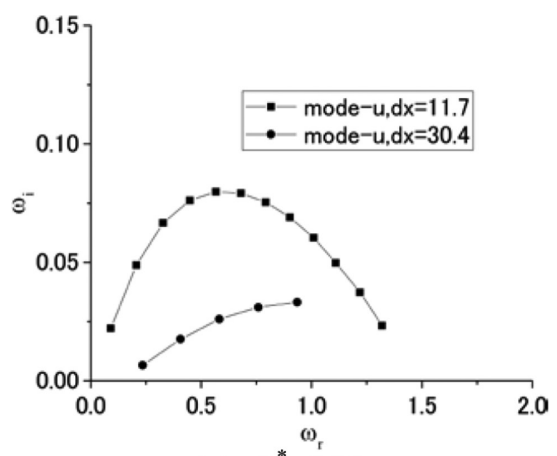

(a) $\Gamma^{*}=6.24$

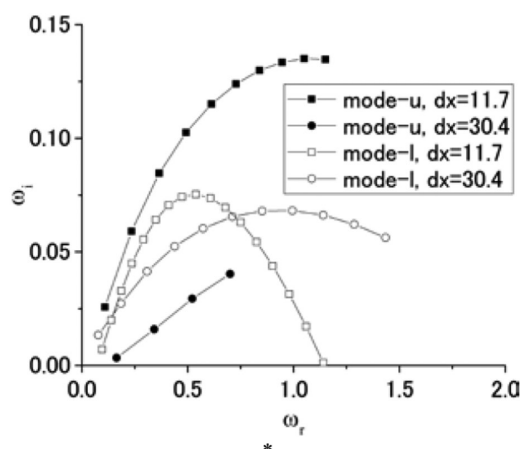

(b) $\Gamma^{*}=12.4$

Figure 4: Distribution of eigenvalues of unstable modes along the variation of $\alpha$ for $\Gamma=6.24$ and $12.4 \beta$ is assumed to be zero.

real part and imaginary part of eigenfunctions of streamwise velocity of mode- $u$ and mode- $l$. Figure 4 shows the distribution of eigenvalues of the unstable modes along the variation of the streamwise wavenumber $\alpha$ for $\Gamma^{*}=6.24$ and 12.4 at $t^{*}=21.75 \beta$ is assumed to be zero.. In the case of $\Gamma^{*}=6.24$, only "mode- $u$ " is observed and amplification at $\mathrm{d} x=11.7$ is larger than that at $\mathrm{d} x=30.4$. As found in Fig. 4b, mode- $u$ is more amplified compared with mode- $l$ at $\mathrm{d} x=11.7$ when $\Gamma^{*}=12.4$, while at $\mathrm{d} x=30.4$, mode- $l$ is more amplified. Comparing the locations of $\mathrm{d} x=11.7$ and $\mathrm{d} x=30.4$, amplification of disturbances at $\mathrm{d} x=11.7$, which is near the root of the vortex tube/upstream, is larger than that at $\mathrm{d} x=30.4$, which is near the head of the vortex tube/downstream. While mode- $u$ amplifies more than mode- $l$ near the upstream side of the vortex tube, mode- $l$ amplifies more near the downstream side of the vortex tube. Thus, the exchange of dominant modes is observed.

Although the formation of the hairpin vortex appears to fall into non-linear stability theory which is different from the present linear stability analysis, the result of the present analysis is compatible with the generation of the secondary hairpin vortices mentioned above. Consistent with the present result shown in Fig. $4 \mathrm{~b}$ where $\omega_{i}$ at $d x=30.4$ is less than $\omega_{i}$ at $\mathrm{d} x=11.7$, downstream hairpin vortices are smaller than upstream vortices observed in Fig. 1 . Although not shown here, maximum amplification takes place when $\alpha=1.8$ for mode- $u$ and $\alpha=2.2$ for mode- $l$ at $\mathrm{d} x=11.7$. This corresponds to the wavelengths $\lambda=3.5 \delta_{\text {in }}$ and $2.10 \delta_{\text {in }}$, respectively. The distance between hairpin vortices appearing in DNS for the corresponding case is around $4.16 \delta_{\text {in }}$ when $t^{*}=108.75$. A distance of even $2.92 \delta_{\text {in }}$ appears in DNS when $t^{*}$ $=152.25$. Therefore, the present analysis predicts the distance between hairpin vortices and disturbances triggering hairpin vortices successfully.

\section{CONCLUSIONS}

Effects of circulation on the evolution of vortex tubes and the associated response of nearwall flows in the shear of laminar boundary-layer flows are investigated by DNS using a model proposed by Hon and Walker. Firstly, dynamics of a single hairpin vortex is investigated. Numerous secondary hairpin vortices, much more than previously reported, which are regularly aligned in the streamwise direction and bridging over the legs, are allowed to be newly generated according to the shear-layer instability of the legs of an initial hairpin vortex. When the circulation is sufficiently large, small-scale turbulence is then produced. 
Secondly, a straight vortex tube model is investigated. Sinuous deformation of a shear layer, which leads to the generation of discrete hairpin vortices, becomes obvious especially near the upper region of the vortex tube. As a result of the quasi-linear stability analysis, it is found that while only one unstable mode appears when the circulation is small, two modes, that, off-wall mode and near-wall mode, appear when the circulation is large. The cases of circulation where the two modes appear correspond to those where the production of smallscale turbulence is observed in the simulations of the single hairpin vortex. Between the neighbourhoods of the tip and head of the straight vortex tube, the exchange of dominant modes is observed.

\section{ACKNOWLEDGEMENTS}

This work was supported by Grant for Basic Science Research Projects from the Sumitomo Foundation and the Institute of Statistical Mathematics (ISM) Cooperative Research Program 2016 ISM-CRP2019. Computational resources are provided by ISM and Japan Aerospace Exploration Agency (JAXA).

\section{REFERENCES}

[1] Smith, C.R., Walker, J.D.A., Haidari, A.H. \& Sobrun, U., On the dynamics of near-wall turbulence. Philosophical Transaction of the Royal Socirty A, 336, pp. 131-175, 1991. http://dx.doi.org/10.1098/rsta.1991.0070

[2] Theodorsen, T., Mechanism of turbulence. Proceeding of Second Midwestern Conference on Fluid Mechanics Bulls, Ohio State University: Columbus, Ohio, 149, 1952.

[3] Lee, C.B. \& Wu, J.Z., Transition in wall-bounded flows. Applied Mechanics Reviews, 61, 2008.

[4] Robinson, S.K., Coherent motions in the turbulent boundary layer. Annual Reviews Fluid Mechanics, 23(601), pp. 601-639, 1991. http://dx.doi.org/10.1146/annurev.fl.23.010191.003125

[5] Robinson, S.K., The kinematics of turbulent boundary layer structure. NASA TM 103859, 1991.

[6] Panton, R., Overview of the self-sustaining mechanisms of wall turbulence. Progress in Aerospace Sciences, 37, pp. 341-383, 2001.

http://dx.doi.org/10.1016/S0376-0421(01)00009-4

[7] Adrian, R.J., Hairpin vortex organization in wall turbulence. Physics of Fluids, 19, 041301, 2007.

http://dx.doi.org/10.1063/1.2717527

[8] Dennis, D.J.C., Coherent structures in wall-bounded turbulence. Annals of the Brazilian Academy of Sciences, 87(2), pp. 1161-1193, 2015.

http://dx.doi.org/10.1590/0001-3765201520140622

[9] Moin, P., Leonard, A. \& Kim, J., Evolution of a curved vortex filament into a vortex ring. Physics of Fluids, 29(4), pp. 955-963, 1986.

http://dx.doi.org/10.1063/1.865690

[10] Acarlar, M. \& Smith, C., A study of hairpin vortices in a laminar boundary layer. Part I. Hairpin vortices generated by a hemisphere protuberance. Journal of Fluid Mechanics, 175, pp. 1-41, 1987.

http://dx.doi.org/10.1017/S0022112087000272

[11] Hon, T.L. \& Walker, J.D.A., Evolution of hairpin vortices in a shear flow. Computers and Fluids, 20(3), pp. 343-358, 1991. 
http://dx.doi.org/10.1016/0045-7930(91)90050-R

[12] Singer, B.A. \& Joslin, R.D., Metamorphosis of a hairpin vortex into a young turbulent spot. Physics of Fluids, 6(11), pp. 3724-3736, 1994.

http://dx.doi.org/10.1063/1.868363

[13] Zhou, J., Adrian, R.J. \& Balachander, S., Autogeneration of near-wall vortical structures in channel flow. Physics of Fluids, 8(1), pp. 288-290, 1996.

http://dx.doi.org/10.1063/1.868838

[14] Zhou, J., Adrian, R.J. \& Balachandar, S., Mechanisms for generating coherent packets of hairpin vortices in channel flow. Journal of Fluid Mechanics, 387, pp. 353-396, 1999.

http://dx.doi.org/10.1017/S002211209900467X

[15] Liu, C. \& Chen, L., Parallel DNS for vortex structure of late stages of flow transition. Computers \& Fluids, 45, pp. 129-137, 2011.

http://dx.doi.org/10.1016/j.compfluid.2010.11.006

[16] Duguet, Y., Schlatter, P., Henningson, D. \& Eckhardt, B., Self-sustained localized structures in a boundary-layer flow. Physics Revised Letters, 108, 044501, 2012.

http://dx.doi.org/10.1103/PhysRevLett.108.044501

[17] Cohen, J., Karp, M. \& Mehta, V., A minimal flow-elements model for the generation of packets of hairpin vortices in shear flows. Journal of Fluid Mechanics, 747, pp. 30-43, 2014. http://dx.doi.org/10.1017/jfm.2014.140

[18] Eitel-Amor, G., Örlu, R., Schlatter, P. \& Flores, O., Hairpin vortices in turbulent boundary layers. Physics of Fluids, 27, 025108, 2015. http://dx.doi.org/10.1063/1.4907783

[19] Sabatino, D.R. \& Maharjan, R., Characterizing the formation and regeneration of hairpin vortices in a laminar boundary layer. Physics of Fluids, 27, 124104, 2015.

[20] Moore, D.W., Finite amplitude waves on aircraft trailing vortices. Aeronautical Quarterly, 23, pp. 307-314, 1972.

[21] Matsuura, K. \& Kato, C., Large-eddy simulation of compressible transitional flows in a low-pressure turbine cascade. AIAA Journal, 45(2), pp. 442-457, 2007. http://dx.doi.org/10.2514/1.22425

[22] Cebechi, T. \& Smith, A.M.O., Analysis of Turbulent Boundary Layer, Academic Press: New York, 1974.

[23] Matsuura, K., Numerical simulation of a straight vortex tube in the shear of a laminar boundary-layer flow. Proceeding of AsiaSim, pp. 1-2, 2015.

[24] Chassaing, P., Antonia, R.A., Anselmet, F., Joly, L. \& Sarkar, S., Variable Density Fluid Turbulence, Kluwer Academic Publishers, 2010.

[25] Malik, M.R., Numerical methods for hypersonic boundary layer stability. Journal of Computational Physics, 86(2), pp. 376-413, 1990.

http://dx.doi.org/10.1016/0021-9991(90)90106-B 